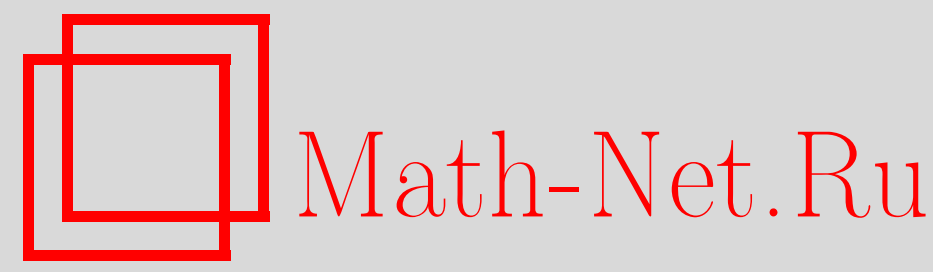

Ю. Г. Зархин, О кольцах эндоморфизмов некоторых якобианов в конечной характеристике, Матем. сб., 2002, том 193, номер 8, 39-48

DOI: https://doi.org/10.4213/sm673

Использование Общероссийского математического портала Math-Net.Ru подразумевает, что вы прочитали и согласны с пользовательским соглашением

http://www . mathnet.ru/rus/agreement

Параметры загрузки:

IP: 54.237 .206 .68

26 апреля 2023 г., 18:00:32 


\title{
Ю.Г. Зархин
}

\section{О кольцах эндоморфизмов некоторых якобианов в конечной характеристике}

\begin{abstract}
Мы доказываем, что при некоторых дополнительных предположениях кольцо всех эндоморфизмов якобиана кривой $y^{\ell}=f(x)$ содержит максимальное коммутативное подкольцо, изоморфное кольцу всех целых алгебраических чисел $\ell$-го кругового поля. Здесь $\ell$ - нечетное простое число, делящее степень $n$ многочлена $f$ и отличное от характеристики основного алгебраически замкнутого поля; при этом $n \geqslant 9$. Дополнительные же предположения состоят в том, что все коэффициенты многочлена $f$ лежат в некотором подполе $K$, над которьм его (многочлена) группа Галуа совпадает либо с полной симметрической группой $S_{n}$, либо со знакопеременной группой $A_{n}$.

Библиограффия: 20 названий.
\end{abstract}

Как обычно, мы используем обозначения $\mathbb{Z}, \mathbb{Q}, \mathbb{C}$ для кольца целых чисел и полей рациональных и комплексных чисел соответственно. Пусть $\ell$ - простое число. Через $\mathbb{F}_{\ell}, \mathbb{Z}_{\ell}, \mathbb{Q}_{\ell}$ мы обозначаем конечное простое поле из $\ell$ элементов, кольцо целых $\ell$-адических чисел и поле $\ell$-адических чисел соответственно. Зафиксируем примитивный корень степени $\ell$ из единищы

$$
\zeta_{\ell} \in \mathbb{C}
$$

Тогда $\mathbb{Q}\left(\zeta_{\ell}\right)$ - $\ell$-е круговое поле, $\mathbb{Z}\left[\zeta_{\ell}\right]$ - его кольцо целых. Хорошо известно, что $\mathbb{Q}\left(\zeta_{\ell}\right)$ - числовое поле степени $\ell-1$.

Пусть $K$ - поле характеристики отличной от $\ell$. Зафиксируем его алгебраическое замькание $K_{a}$ и обозначим через $\mathrm{Gal}(K)$ его абсолютную группу Галуа $\operatorname{Aut}\left(K_{a} / K\right)$. Зафиксируем также примитивный корень степени $\ell$ из единицы

$$
\zeta \in K_{a}
$$

Пусть $f(x) \in K[x]$ - многочлен степени $n \geqslant 4$ без кратных корней. Далее мы предполагаем, что либо $\operatorname{char}(K)=0$, либо $\ell \mid n$.

Пусть $C_{f, \ell}-$ гладкая проективная модель гладкой аффинной кривой

$$
y^{\ell}=f(x)
$$

Хорошо известно [1; с. 401, 402], [2; предложение 1, с. 3359], [3; с. 148], что род $g\left(C_{f, \ell}\right)$ кривой $C_{f, \ell}$ равен $(\ell-1)(n-1) / 2$, если $\ell$ не делит $n$, и $(\ell-1)(n-2) / 2$, если делит.

Отображение

$$
(x, y) \mapsto(x, \zeta y)
$$

Работа вьполнена при поддержке Национального научного фонда (NSF) CША. 
задает нетривиальный бирациональный автоморфизм

$$
\delta_{\ell}: C_{f, \ell} \rightarrow C_{f, \ell}
$$

периода $\ell$.

Пусть $J^{(f, \ell)}=J\left(C_{f, \ell}\right)$ - якобиан кривой $C_{f, \ell}$; это абелево многообразие размерности $g\left(C_{f, \ell}\right)$. Обозначим через $\operatorname{End}\left(J^{(f, \ell)}\right)$ кольцо всех $K_{a}$-эндоморфизмов абелева многообразия $J^{(f, \ell)}$. В силу функториальности $\delta_{\ell}$ индуцирует автоморфизм абелева многообразия $J^{(f, \ell)}$, который мы по-прежнему обозначаем через $\delta_{\ell}$. Известно [3; с. 149], [4; с. 448], что

$$
\delta_{\ell}^{\ell-1}+\cdots+\delta_{\ell}+1=0
$$

в $\operatorname{End}\left(J^{(f, \ell)}\right)$, и это дает нам естественное вложение

$$
\mathbb{Z}\left[\zeta_{\ell}\right] \cong \mathbb{Z}\left[\delta_{\ell}\right] \subset \operatorname{End}\left(J^{(f, \ell)}\right)
$$

при котором $\zeta_{\ell}$ переходит в $\delta_{\ell}$.

Основным результатом настоящей статьи является следуюшее утверждение.

Теорема 0.1. Пусть $K$ - поле характеристики отличной от $\ell$. Пусть $n \geqslant 9$ - натуральное число, делящееся на $\ell$. Предположим, что $f(x) \in K[x]$ - неприводимый многочлен степени $n$ и его группа Галуа совпадает либо $c$ полной симметрической группой $S_{n}$, либо со знакопеременной группой $A_{n}$.

Тогда $\mathbb{Z}\left[\delta_{\ell}\right]$ - максимальное коммутативное подкольцо в $\operatorname{End}\left(J^{(f, \ell)}\right)$. Другими словами, если эндоморфизм якобиана $J^{(f, \ell)}$ коммутирует с автоморфизмом $\delta_{\ell}$, то он лежит в $\mathbb{Z}\left[\delta_{\ell}\right]$.

ЗАмечАниЕ 0.2. Случай $\ell=2$ (т.е. случай гиперэллиптических кривых, для которых, как хорошо известно, $\delta_{2}=-1$ ) рассмотрен в [5], [6] (см. также [7], [8]). Ясно, что $\mathbb{Z}\left[\delta_{2}\right]=\mathbb{Z}$, и утверждение теоремы означает, что $\operatorname{End}\left(J^{(f, 2)}\right)=\mathbb{Z}$.

Для полей $K$ нулевой характеристики случай нечетного $\ell$ рассмотрен в [9]; см. также [10], где доказано, что $\operatorname{End}\left(J^{(f, \ell)}\right)=\mathbb{Z}\left[\delta_{\ell}\right]$ для всех $n \geqslant 5$.

Тем самым, при доказательстве теоремы 0.1 достаточно ограничиться случаем, ког да $\ell$ нечетно и делит $n, \operatorname{a~} \operatorname{char}(K)>0$.

Мы выведем теорему 0.1 из вспомогательного утверждения (теорема 0.3), представляющего некоторый самостоятельньй интерес. Но вначале обсудим следующую ситуацию.

Пусть $X$ - абелево многообразие над $K$ и $\operatorname{End}(X)$ - кольцо всех его $K_{a}$-эндоморфизмов. Обозначим через $\operatorname{End}_{K}(X)$ подкольцо в $\operatorname{End}(X)$, состоящее из всех эндоморфизмов, определенных над $K$. Пусть $E$ - числовое поле, $\mathscr{O}=\mathscr{O}_{E} \subset E$ - его кольцо целых, $\lambda$-максимальный идеал в $\mathscr{O}$ такой, что соответствующее (конечное) поле вычетов $k(\lambda)=\mathscr{O} / \lambda$ имеет нечетную характеристику $\ell$. Предположим, что задано вложение

$$
\mathscr{O} \hookrightarrow \operatorname{End}_{K}(X) \subset \operatorname{End}(X)
$$

переводящее $1 \in \mathscr{O}$ в тождественный эндоморфизм. Далее мы отождествляем $\mathscr{O}$ с его образом в $\operatorname{End}(X)$. Хорошо известно [11], [12], что

$$
d=\frac{2 \operatorname{dim}(X)}{[E: \mathbb{Q}]}
$$


- положительное целое число. Рассмотрим коммутативную группу

$$
X_{\lambda}=\left\{x \in X\left(K_{a}\right): a x=0 \forall a \in \lambda \subset \mathscr{O} \subset \operatorname{End}(X)\right\} .
$$

Легко видеть, что $X_{\lambda}-k(\lambda)$-векторное пространство; известно [13; $\S 7.6$, предложение 7.20], что его размерность равна $d$. С другой стороны, так как $\mathscr{O} \subset \operatorname{End}_{K}(X)$, подгруппа

$$
X_{\lambda} \subset X\left(K_{a}\right)
$$

Галуа-инвариантна и действие на нее групшы $\mathrm{Gal}(K)$ задает естественный гомоморфизм

$$
\bar{\rho}_{\lambda, X}: \operatorname{Gal}(K) \rightarrow \operatorname{Aut}_{k(\lambda)}\left(X_{\lambda}\right) \cong \mathrm{GL}_{d}(k(\lambda))
$$

Чтобы описать этот гомоморфизм, предположим для простоты, что $\ell \mathscr{O}=\lambda^{[E: \mathbb{Q}]}$, т.е. $\lambda$ - единственный максимальный идеал в $\mathscr{O}$, лежащий над $\ell$, и $k(\lambda)=\mathbb{F} \ell$. Тогда

$$
\mathscr{O} \otimes \mathbb{Z}_{\ell}=\mathscr{O}_{\lambda}
$$

где $\mathscr{O}_{\lambda}$ - пополнение кольца $\mathscr{O}$ в $\lambda$-адической топологии. Хорошо известно, что $\mathscr{O}_{\lambda}$ - кольцо главных идеалов с единственным максимальньм идеалом $\lambda \mathscr{O}_{\lambda}$. Легко видеть, что

$$
\ell \mathscr{O}_{\lambda}=\left(\lambda \mathscr{O}_{\lambda}\right)^{[E: \mathbb{Q}]}
$$

Зафиксируем элемент $c$ идеала $\lambda$, не лежаший в $\lambda^{2}$. Легко видеть, что $\lambda \mathscr{O}_{\lambda}=c \mathscr{O}_{\lambda}$. Отсюда вытекает, что найдется единица $u \in \mathscr{O}_{\lambda}^{*}$ такая, что

$$
\ell=u c^{[E: \mathbb{Q}]} \text {. }
$$

Также из однозначности разложения для идеалов в $\mathscr{O}$ следует, что

$$
\lambda=\ell \mathscr{O}+c \mathscr{O} \text {. }
$$

Отсюда немедленно вытекает, что

$$
X_{\lambda}=\left\{x \in X_{\ell}: c x=0\right\} \subset X_{\ell} .
$$

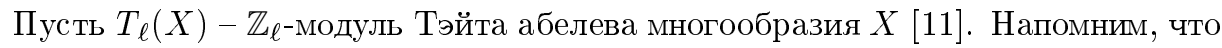

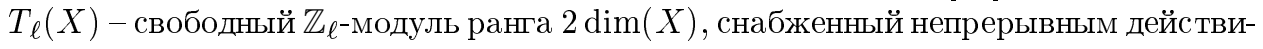
em

$$
\rho_{\ell, X}: \operatorname{Gal}(K) \rightarrow \operatorname{Aut}_{\mathbb{Z}_{\ell}}\left(T_{\ell}(X)\right) \cong \operatorname{GL}_{2 \operatorname{dim}(X)}\left(\mathbb{Z}_{\ell}\right)
$$

и естественньм вложением

$$
\operatorname{End}_{K}(X) \otimes \mathbb{Z}_{\ell} \hookrightarrow \operatorname{End}_{\mathbb{Z}_{\ell}}\left(T_{\ell}(X)\right)
$$

образ которого коммутирует с $\rho_{\ell, X}(\operatorname{Gal}(K)) \subset \operatorname{Aut}_{\mathbb{Z}_{\ell}}\left(T_{\ell}(X)\right)$. Также имеется естественный изоморфизм модулей Галуа

$$
X_{\ell}=T_{\ell}(X) / \ell T_{\ell}(X)
$$

коммутирующий с естественными действиями кольца $\operatorname{End}_{K}(X) \otimes \mathbb{Z}_{\ell} \supset \mathscr{O}$ на $T_{\ell}(X)$ и $X_{\ell}$. Отсюда вытекает, что $\mathscr{O}[\mathrm{Gal}(K)]$-модуль $X_{\lambda}$ равен

$$
\begin{aligned}
c^{-1} \ell T_{\ell}(X) / \ell T_{\ell}(X) & =c^{[E: \mathbb{Q}]-1} \ell T_{\ell}(X) / c^{[E: \mathbb{Q}]} T_{\ell}(X) \cong T_{\ell}(X) / c T_{\ell}(X) \\
& =T_{\ell}(X) / \lambda T_{\ell}(X)=T_{\ell}(X) / \lambda \mathscr{O}_{\lambda} T_{\ell}(X) .
\end{aligned}
$$

Тем самым,

$$
X_{\lambda} \cong T_{\ell}(X) / c T_{\ell}(X)=T_{\ell}(X) / \lambda T_{\ell}(X)=T_{\ell}(X) /\left(\lambda \mathscr{O}_{\lambda}\right) T_{\ell}(X)
$$


ТЕОРема 0.3. Пусть $\ell-$ простое число, $K$ - поле характеристики отличной от $\ell$. Зафиксируем алгебраическое замыкание $K_{a}$ поля $K$ и обозначим через $\operatorname{Gal}(K)$ его абсолютную группу Галуа $\operatorname{Aut}\left(K_{a} / K\right)$. Пусть $X$ - абелево многообразие над $K u \operatorname{End}(X)$ - кольцо всех его $K_{a}$-эндоморфизмов. Обозначим через $\operatorname{End}_{K}(X)$ подкольцо в $\operatorname{End}(X)$, состоящее из всех эндоморфизмов, определенных над $K$. Пусть $E$ - числовое поле, $\mathscr{O}=\mathscr{O}_{E} \subset E-$ его кольцо цельих, $\lambda$-максимальный идеал в $\mathscr{O}$ такой, что соответствующее (конечное) поле вычетов $k(\lambda)=\mathscr{O} / \lambda$ имеет нечетную характеристику $\ell$. Предположим, что $\lambda$-единственньй максимальный идеал в $\mathscr{O}$, лежсащий над $\ell, u$ $k(\lambda)=\mathbb{F}_{\ell}$.

Предположим, что задано вложение

$$
\mathscr{O} \hookrightarrow \operatorname{End}_{K}(X) \subset \operatorname{End}(X)
$$

переводящее $1 \in \mathscr{O}$ в тождественный эндоморфизм. Далее мй отождествляем $\mathscr{O}$ с его образом в $\operatorname{End}(X)$. Положим

$$
d=\frac{2 \operatorname{dim}(X)}{[E: \mathbb{Q}]}
$$

и рассмотрим d-мерное векторное пространство $X_{\lambda}$ над $k(\lambda)$.

Обозначим через $\widetilde{G}_{\lambda, X}$ образ естественного гомоморфизма

$$
\bar{\rho}_{\lambda, X}: \operatorname{Gal}(K) \rightarrow \operatorname{Aut}_{k(\lambda)}\left(X_{\lambda}\right) .
$$

Предположим, что $d \geqslant 7, \ell \mid(d+2)$ и $\widetilde{G}_{\lambda, X}$ содержит подгруппу, изоморфную $A_{d+2}$.

Тогда $\mathscr{O}$ - максимальное коммутативное подкольцо в $\operatorname{End}(X)$. Другими словами, если эндоморфизм абелева многообразия $X$ коммутирует с $\mathscr{O}$, то он сам лежсти в $\mathscr{O}$.

Мы выведем теорему 0.1 из теоремы 0.3 в $\S 1$. Теорема 0.3 будет доказана в $\S 4$. Параграфы 2 и 3 содержат вспомогательные результаты из теории представлений, используемые при доказательстве теоремы 0.3 .

\section{§1. Доказательство теоремы 0.1}

В этом параграфе мы докажем теорему 0.1, предполагая справедливость теоремы 0.3 .

Мы предполагаем, что $\ell$ нечетно и делит $n$. Заменив (в случае $\operatorname{Gal}(f)=S_{n}$ ) поле $K$ на соответствуюшее квадратичное расширение, мы можем считать, что $\operatorname{Gal}(f)=A_{n}$. Так как группа Галуа $\operatorname{Gal}(K(\zeta) / K)$ абелева, а группа $A_{n}$ совершенна, то, заменив $K$ на $K(\zeta)$, мы можем считать, что $\zeta \in K$.

Положим $X=J^{(f, \ell)}$. Так как $\zeta \in K$, то $\delta_{\ell} \in \operatorname{End}_{K}(X)$. Положим

$$
E=\mathbb{Q}\left(\zeta_{\ell}\right), \quad \mathscr{O}=\mathbb{Z}\left[\zeta_{\ell}\right] \cong \mathbb{Z}\left[\delta_{\ell}\right] \subset \operatorname{End}_{K}\left(J^{(f, \ell)}\right) \subset \operatorname{End}\left(J^{(f, \ell)}\right) .
$$

Имеем

$$
d=\frac{2 \operatorname{dim}\left(J^{(f, \ell)}\right)}{\left[\mathbb{Q}\left(\zeta_{\ell}\right): \mathbb{Q}\right]}=\frac{(\ell-1)(n-2)}{\ell-1}=n-2 .
$$

Мы получаем, что $d \geqslant 7$ и $d+2=n$ делится на $\ell$. 
Обозначим через $\lambda$ максимальньй идеал

$$
\left(1-\zeta_{\ell}\right) \mathbb{Z}\left[\zeta_{\ell}\right]=\left(1-\delta_{\ell}\right) \mathbb{Z}\left[\delta_{\ell}\right]
$$

Имеем

$$
k(\lambda)=\mathbb{Z}\left[\zeta_{\ell}\right] /\left(1-\zeta_{\ell}\right) \mathbb{Z}\left[\zeta_{\ell}\right]=\mathbb{F}_{\ell}
$$

Дадим теперь описание модуля Галуа $J_{\lambda}^{(f, \ell)}$, следуя [3]. Пусть $R=\left\{a_{1}, \ldots, a_{n}\right\} \subset$ $K_{a}$ - множество всех корней многочлена $f$. Мы можем рассматривать группу $S_{n}$ как группу всех перестановок множества $R$. Группа Галуа $\operatorname{Gal}(f)$ многочлена $f$ переставляет эти корни и тем самьм реализуется как подгруппа в $S_{n}$. Напомним также, что определена каноническая сюръекция

$$
\operatorname{Gal}(K) \rightarrow \operatorname{Gal}(f)
$$

Действие группы $\mathrm{Gal}(f)$ на множестве $R$ задает стандартное перестановочное представление в $n$-мерном $\mathbb{F}_{\ell}$-векторном пространстве $\mathbb{F}_{\ell}^{R}$ всех функций $\psi: R \rightarrow \mathbb{F} \ell$. Это представление не является неприводимьм. Действительно, “прямая" постоянных функций $\mathbb{F}_{\ell} \cdot 1$ и гиперплоскость $\left(\mathbb{F}_{\ell}^{B}\right)^{0}:=\left\{\psi: \sum_{i=1}^{n} \psi\left(a_{i}\right)=0\right\}$ являются $\operatorname{Gal}(f)$-инвариантными подпространствами в $\mathbb{F}_{\ell}^{R}$. Так как $\ell \mid n$, гиперплоскость $\left(\mathbb{F}_{\ell}^{B}\right)^{0}$ содержит прямую $\mathbb{F}_{\ell} \cdot 1$, и мы получаем естественное точное представление групшы $\mathrm{Gal}(f)$ в $(n-2)$-мерном $\mathbb{F}_{\ell}$-векторном факторпространстве

$$
\left(\mathbb{F}_{\ell}^{B}\right)^{00}:=\left(\mathbb{F}_{\ell}^{B}\right)^{0} /\left(\mathbb{F}_{\ell} \cdot 1\right),
$$

называемом сердечником (heart) перестановочного действия группы $\operatorname{Gal}(f)$ на множество $R$.

Каноническая сюръекция $\operatorname{Gal}(K) \rightarrow \operatorname{Gal}(f)$ задает на точном $\operatorname{Gal}(f)$-модуле $\left(\mathbb{F}_{\ell}^{B}\right)^{00}$ естественную структуру $\mathrm{Gal}(K)$-модуля. Известно [3; предложение 6.2], что $\operatorname{Gal}(K)$-модули $\left(\mathbb{F}_{\ell}^{B}\right)^{00}$ и $J_{\lambda}^{(f, \ell)}$ канонически изоморфны. Отсюда, в частности, вытекает, что $\widetilde{G}_{\lambda, X} \cong \operatorname{Gal}(f)$ для $X=J^{(f, \ell)} \cdot \operatorname{Bcпоминая,~что~} \operatorname{Gal}(f)=A_{n}$, мы получаем, что $\widetilde{G}_{\lambda, X} \cong A_{n}=A_{d+2}$. Теперь теорема 0.1 легко следует из теоремы 0.3, примененной к $X=J^{(f, \ell)}, \mathscr{O}=\mathbb{Z}\left[\delta_{\ell}\right]$ и $\lambda=\left(1-\delta_{\ell}\right) \mathbb{Z}\left[\delta_{\ell}\right]$.

\section{§ 2. Очень простые представления}

Следующее понятие введено автором в [7].

ОПРЕДЕЛЕНИЕ 2.1. Пусть $V$ - векторное пространство над полем $k, G$ - группа и $\rho: G \rightarrow \operatorname{Aut}_{k}(V)$ - линейное представление группы $G$ в пространстве $V$. Мы говорим, что $G$-модуль $V$ очень $п р о с т$, если он удовлетворяет следуюшему условию:

если $R \subset \operatorname{End}_{k}(V)-k$-подалгебра, содержашая тождественный оператор Id, такая, что

$$
\rho(\sigma) R \rho(\sigma)^{-1} \subset R \quad \forall \sigma \in G
$$

то либо $R=k \cdot \mathrm{Id}$, либо $R=\operatorname{End}_{k}(V)$.

Это условие очевидным образом эквивалентно следующему:

если $R \subset \operatorname{End}_{k}(V)-k$-подалгебра, содержащая тождественный оператор Id и инвариантная относительно сопряжений с помощью $\rho(\sigma)$ (для всех $\sigma \in G)$, то либо $\operatorname{dim}_{k}(R)=1$, либо $\operatorname{dim}_{k}(R)=\left(\operatorname{dim}_{k}(V)\right)^{2}$. 
ЗАмечАнИЯ 2.2. (i) Ясно, что $G$-модуль $V$ очень прост, если и только если соответствующий $\rho(G)$-модуль $V$ очень прост.

(ii) Ясно, что если $V$ очень прост, то соответствующий гомоморфизм $k$-алгебр

$$
k[G] \rightarrow \operatorname{End}_{k}(V)
$$

сюръективен. Здесь $k[G]$ - групповая $k$-алгебра группы $G$. В частности, очень простой модуль абсолютно прост.

(iii) Если $G^{\prime}$ - подгруппа в $G$ и соответствующий $G^{\prime}$-модуль $V$ очень прост, то $G$-модуль $V$ также очень прост.

(iv) Пусть $G^{\prime}$ - нормальная подгруппа в $G$. Если $V$ - очень простой $G$-модуль, то либо $\rho\left(G^{\prime}\right) \subset \operatorname{Aut}_{k}(V)$ состоит из скаляров (т.е. лежит в $\left.k \cdot \mathrm{Id}\right)$, либо соответствующий $G^{\prime}$-модуль $V$ абсолютно прост. Действительно, пусть $R^{\prime} \subset \operatorname{End}_{k}(V)-$ образ естественного гомоморфизма $k\left[G^{\prime}\right] \rightarrow \operatorname{End}_{k}(V)$. Ясно, что $R^{\prime}$ инвариантен относительно сопряжения элементами из $G$. Значит, либо $R^{\prime}$ состоит из скаляров и, следовательно, $\rho\left(G^{\prime}\right) \subset R^{\prime}$ состоит из скаляров, либо $R^{\prime}=\operatorname{End}_{k}(V)$ и, следовательно, $G^{\prime}$-модуль $V$ абсолютно прост.

(v) Пусть $O_{F}$ - кольцо дискретного нормирования с полем частных $F$, максимальным идеалом $m_{F}$ и полем вычетов $k=O_{F} / m_{F}$. Пусть $V_{F}$ - конечномерное векторное пространство над $F$,

$$
\rho_{F}: G \rightarrow \operatorname{Aut}_{F}\left(V_{F}\right)
$$

- $F$-линейное представление группы $G$. Пусть $T-G$-инвариантная $O_{F}$-решетка в $V_{F}$ и соответствующий $k[G]$-модуль $T / m_{F} T$ изоморфен $V$. Предположим, что $G$-модуль $V$ очень прост. Тогда $G$-модуль $V_{F}$ также очень прост. Другими словами, подгем очень простого представления также очень прост. Действительно, пусть $R_{F} \subset \operatorname{End}_{F}\left(V_{F}\right)-F$-подалгебра, содержашая тождественный оператор и инвариантная относительно сопряжений с помощью $\rho_{F}(\sigma)$ (для всех $\sigma \in G$ ). Положим

$$
R_{O}=R \cap \operatorname{End}_{O_{F}}(T) \subset \operatorname{End}_{O_{F}}(T) .
$$

Ясно, что $R_{O}-$ свободньй $O_{F}$-модуль, ранг которого совпадает с $\operatorname{dim}_{F}\left(R_{F}\right)$. Также ясно, что $R_{O}$ - чистый $O_{F}$-подмодуль в $\operatorname{End}_{O_{F}}(T)$, т.е. $O_{F}$-фактормодуль $\operatorname{End}_{O_{F}}(T) / R_{O}$ не имеет кручения. Положим

$R_{k}=R_{O} / m_{F} R_{O}=R_{O} \otimes_{O_{F}} k \subset \operatorname{End}_{O_{F}}(T) \otimes_{O_{F}} k=\operatorname{End}_{k}\left(T / m_{F} T\right)=\operatorname{End}_{k}(V)$.

Ясно, что $R_{k}$ является $k$-подалгеброй в $\operatorname{End}_{k}(V)$ размерности $\operatorname{dim}_{F}\left(R_{F}\right)$, содержит тождественный оператор и инвариантна относительно сопряжений с помощью элементов $G$. Теперь то, что $V$ очень прост, влечет за собой то, что либо $\operatorname{dim}_{k}\left(R_{k}\right)=1$, либо $\operatorname{dim}_{k}\left(R_{k}\right)=\operatorname{dim}_{k}(V)^{2}$. Поскольку $\operatorname{dim}_{k}(V)=\operatorname{dim}_{F}\left(V_{F}\right)$, мы получаем, что либо $\operatorname{dim}_{F}\left(R_{F}\right)=1$, либо $\operatorname{dim}_{F}\left(R_{F}\right)=\operatorname{dim}_{F}\left(V_{F}\right)^{2}$. Ясно, что в первом случае $R_{F}$ состоит из скаляров, а во втором $R_{F}=\operatorname{End}_{F}\left(V_{F}\right)$.

ПримеР 2.3. Пусть $n>6$ - натуральное число, $W$ - векторное пространство над $\mathbb{F}_{p}$, размерность которого равна $n-1$, если $p$ не делит $n$, и равна $n-2$, если $p \mid n$. Если $p=2$, то мы дополнительно предполагаем, что $n>8$. Предположим, что задано вложение

$$
A_{n} \hookrightarrow \operatorname{Aut}_{\mathbb{F}_{p}}(W)
$$


Тогда $A_{n}$-модуль $W$ очень прост. Действительно, положим $n^{\prime}=\operatorname{dim}_{\mathbb{F}_{p}}(W)$. Известно [14], [15], что точное $n^{\prime}$-мерное представление группы $A_{n}$ над $\mathbb{F}_{p}$ единственно с точностью до изоморфизма. С другой стороны, известно [9; теорема 4.7], что некоторое специальное представление такого вида очень просто. Отсюда вытекает, что любое точное $n^{\prime}$-мерное представление группы $A_{n}$ над $\mathbb{F}_{p}$ (и, в частности, $W$ ) очень просто.

\section{§3. Проективные представления знакопеременной группы}

Пусть $n \geqslant 8$ - натуральное число. Рассмотрим нерасшепляющуюся короткую точную последовательность

$$
1 \rightarrow \mathbb{Z} / 2 \mathbb{Z} \hookrightarrow A_{n}^{\prime} \rightarrow A_{n} \rightarrow 1,
$$

задающую нетривиальное центральное расширение группы $A_{n}$ (т.е. $A_{n}^{\prime}$ - универсальное центральное расширение совершенной групшы $\left.A_{n}\right)$.

ПрЕДЛОЖЕНИЕ 3.1. Любое точное линейное представление группы $A_{n}^{\prime}$ в характеристике 0 имеет размерность $>n-2$.

ДокАЗАТЕльство. Если $8 \leqslant n \leqslant 14$, то наше утверждение следует из таблицы характеров [16]. Поэтому далее мы предполагаем, что $n \geqslant 15$.

Рассмотрим двоичное представление

$$
n=2^{w_{1}}+\cdots+2^{w_{s}}
$$

числа $n$. Здесь $w_{i}$ - различные неотрицательные целые числа, $w_{1}<\cdots<w_{s}$, a $s-$ точное число ненулевых разрядов в двоичной записи числа $n$. Легко видеть, что каждое $w_{i} \geqslant i-1$ и, следовательно, $n \geqslant 2^{s}-1$. Отсюда вытекает, что

$$
s \leqslant \log _{2}(n+1) .
$$

По теореме Вагнера [17; с. 583, 584, теорема 1.3(ii)] каждое точное линейное представление группы $A_{n}^{\prime}$ в характеристике $\neq 2$ имеет размерность, делящуюся на $N:=2^{\left\lfloor\frac{n-s-1}{2}\right\rfloor}$. Стало быть, нам достаточно убедиться в том, что $n-2<N$ для всех $n \geqslant 15$.

Если $n \geqslant 15$, то $2^{n-2}>(n+1)(n-2)^{2}$. Тогда $2^{n-2-\log _{2}(n+1)}>(n-2)^{2}$ и, следовательно, $2^{n-s-2}>(n-2)^{2}$. Извлекая квадратные корни из обеих частей последнего неравенства, мы получаем $2^{\frac{n-s-2}{2}}>n-2$. Отсюда легко вытекает, что $N=2^{\left\lfloor\frac{n-s-1}{2}\right\rfloor}>n-2$.

СлЕДСТВИЕ 3.2. Пусть $n \geqslant 8$ - натуральное число, $\gamma: H \rightarrow A_{n}$ - сюрвективный гомоморфизм конечных групп. Тогда любое точное представление группь $H$ в характеристике 0 имеет размерность $>n-2$.

ДокАЗАТЕльство. Заметим вначале, что любое нетривиальное проективное представление группы $A_{n}$ в характеристике 0 имеет размерность $>n-2$. Действительно, любое нетривиальное проективное представление группы $A_{n}$ есть "проективизация" точного линейного представления либо групшы $A_{n}$, либо групшы $A_{n}^{\prime}$. Остается напомнить, что все точные представления груп $A_{n}$ и $A_{n}^{\prime}$ в характеристике 0 имеют размерность $>n-2$. Для группы $A_{n}^{\prime}$ это утверждение доказано в предложении 3.1 , а для представлений групшы $A_{n}$ оно хорошо известно $[18 ;$ с. 71 , теорема 2.5.15]. 
Теперь предположим, что утверждение доказываемого нами следствия неверно, т.е. для некоторого поля $F$ нулевой характеристики существует вложение $H \hookrightarrow \mathrm{GL}(n-2, F)$. Далее, мы будем отождествлять группу $H$ с его образом в $\mathrm{GL}(n-2, F)$. Заменив поле $F$ на его алгебраическое замыкание, мы можем (и будем) считать $F$ алгебраически замкнутым. Пусть $Z$ - пересечение группы $H$ с подгруппой всех скалярных матриц в $\mathrm{GL}(n-2, F)$. Ясно, что $Z$ - центральная подгруппа в $H$ и

$$
H / Z \subset \operatorname{PGL}(n-2, F) .
$$

$\mathrm{C}$ другой стороны, из простоты и неабелевости группы $A_{n}$ вытекает, что $Z \subset$ $\operatorname{ker}(\gamma)$ и, следовательно, существует сюръекция

$$
H / Z \rightarrow A_{n}
$$

Теперь из теоремы Фейта-Титса (см. [19], а также [20; с. 315, 316]) вытекает существование вложения $A_{n} \hookrightarrow \mathrm{PGL}(n-2, F)$, что противоречит отсутствию нетривиальных проективных представлений групшы $A_{n}$ в характеристике 0 размерности $\leqslant n-2$.

ТЕОРема 3.3. Пусть $\ell$ - нечетное простое число, $F$ - дискретно нормированное поле нулевой характеристики с кольцом нормирования $\mathscr{O}_{F}$, максимальным идеалом $\mathfrak{m}_{F}$ и полем вичетов $\mathscr{O}_{F} / \mathfrak{m}_{F}=\mathbb{F}_{\ell}$. Пусть $d \geqslant 6$ - иелое число такое, что $\ell \mid(d+2)$. Пусть $V-d$-мерное векторное пространство над $F, \Lambda$ - $\mathscr{O}_{F}$-решетка в $V, W:=\Lambda / \mathfrak{m}_{F} \Lambda$ - d-мерное векторное пространство над $\mathbb{F}_{\ell}$. Пусть

$$
G \subset \operatorname{Aut}_{\mathscr{O}_{F}}(\Lambda) \subset \operatorname{Aut}_{F}(V)
$$

- әруппа такая, что ее образ

$$
\widetilde{G} \subset \operatorname{Aut}_{\mathbb{F}_{\ell}}(W)
$$

относительно естественного гомоморфизма редукиии по модулю $\mathfrak{m}_{F}$

$$
\operatorname{red}: \operatorname{Aut}_{\mathscr{O}_{F}}(\Lambda) \hookrightarrow \operatorname{Aut}_{\mathbb{F}_{\ell}}(W)
$$

содержит подгруппу, изоморфную $A_{d+2}$. Тогда

(i) группа $G$ бесконечна;

(ii) для любой подгруппь $G^{\prime}$ конечного индекса в $G$ естественное представление группь $G^{\prime}$ в $F$-пространстве $V$ абсолютно неприводимо.

ДокАЗАтЕльство. Заменив $G$ на подходящую подгруппу, мы можем считать, что $\widetilde{G}=A_{d+2}$. Из простоты и некоммутативности группы $A_{d+2}$ вытекает, что, заменив $G$ на ее пересечение со специальной линейной группой $\mathrm{SL}_{\mathscr{O}}(\Lambda)$, мы можем считать, что

$$
G \subset \mathrm{SL}_{\mathscr{O}}(\Lambda) \subset \mathrm{SL}_{F}(W)
$$

Из примера 2.3 вытекает, что $\widetilde{G}\left(=A_{d+2}\right)$-модуль $W$ очень прост, а из замечания $2.2(\mathrm{v})$ - что $G$-модуль $V$ также очень прост. В частности, согласно замечанию 2.2 (iі) естественное представление группы $G$ в $F$-пространстве $V$ абсолютно неприводимо. Обозначим через $G_{0}$ нормальную подгруппу в $G$, являющуюся ядром отображения редукции

$$
\text { red: } G \rightarrow \widetilde{G} \subset \operatorname{Aut}_{\mathbb{F}_{\ell}}(W) .
$$


Если $G_{0}=\{1\}$, то $G \cong \widetilde{G}=A_{d+2}$ и, следовательно, $V-d$-мерное пространство точного представления групшы $A_{d+2}$, что невозможно ввиду предложения 3.1 . Следовательно, $G_{0} \neq\{1\}$.

Если $G_{0}$ - центральная подгруппа в $G$, то из абсолютной неприводимости $F[G]$-модуля $V$ вытекает, что $G_{0}$ состоит из скаляров. Принимая во внимание естественный изоморфизм

$$
G / G_{0} \cong \widetilde{G}=A_{d+2},
$$

мы получаем, что вложение $G \subset \operatorname{Aut}_{F}(V)$ задает нетривиальное проективное представление групшы $A_{d+2}$ в $d$-мерном пространстве, что невозможно ввиду предложения 3.1. Следовательно, $G_{0}$ не является центральной подгруппой в $G$. В частности, $G_{0}$ не является циклической группой из двух элементов. Отметим также, что ввиду простоты и неабелевости групшы $G / G_{0}=\widetilde{G}=A_{d+2}$ центр групшы $G$ лежит в $G_{0}$.

Предположим, что группа $G_{0}$ конечна, т.е. $G$ конечна. Имеем

$$
G \subset \operatorname{Aut}_{F}(V) \cong \mathrm{GL}_{d}(F)
$$

Применяя следствие 3.2 к $n=d+2$ и $H=G$, мы получаем противоречие, которое доказывает бесконечность групшы $G$ и, тем самым, утверждение (i) теоремы 3.3.

Для доказательства утверждения (ii) достаточно ограничиться случаем нормальной подгруппы $G^{\prime}$. Из бесконечности $G$ и конечности индекса $\left[G: G^{\prime}\right]$ вытекает бесконечность групшы $G^{\prime}$. Поскольку $G^{\prime} \subset G \subset \mathrm{SL}(V)$, бесконечная подгруппа $G^{\prime}$ не может состоять из скаляров. С другой стороны, то, что $G$-модуль $V$ очень прост, влечет за собой согласно замечанию 2.2 (iv) либо абсолютную простоту $G^{\prime}$-модуля $V$, либо включение $G^{\prime} \subset F \cdot \mathrm{Id}$.

\section{§4. Доказательство теоремы 0.3}

Мы выведем теорему 0.3 из теоремы 3.3. Для этого положим

$$
F=E_{\lambda}, \quad \mathscr{O}_{F}=\mathscr{O}_{\lambda}, \quad m_{F}=\lambda \mathscr{O}_{\lambda}, \quad V=V_{\ell}(X), \quad \Lambda=T_{\ell}(X), \quad W=X_{\lambda} .
$$

Положим также

$$
G:=\rho_{\ell, X}(\operatorname{Gal}(K)) \subset \operatorname{Aut}_{\mathscr{O}_{\lambda}}\left(T_{\ell}(X)\right) \subset \operatorname{Aut}_{\mathbb{Z}_{\ell}}\left(T_{\ell}(X)\right)
$$

Сушествует конечное расширение Галуа поля $K$, над которым определены все эндоморфизмы абелева многообразия $X$. Положим

$$
G^{\prime}:=\rho_{\ell, X}\left(\operatorname{Gal}\left(K^{\prime}\right)\right) \subset \operatorname{Aut}_{\mathscr{O}_{\lambda}}\left(T_{\ell}(X)\right) \subset \operatorname{Aut}_{\mathbb{Z}_{\ell}}\left(T_{\ell}(X)\right)
$$

Ясно, что $G^{\prime}$ - нормальная подгруппа конечного индекса в группе $G$, коммутирующая c

$$
\operatorname{End}(X) \otimes \mathbb{Z}_{\ell}=\operatorname{End}_{K^{\prime}}(X) \otimes \mathbb{Z}_{\ell} \subset \operatorname{End}_{\mathbb{Z}_{\ell}}\left(T_{\ell}(X)\right)
$$

Применяя теорему 3.3, мы находим, что $E_{\lambda}\left[G^{\prime}\right]$-модуль $V_{\ell}(X)$ абсолютно прост. Иначе говоря, любой эндоморфизм векторного $\mathbb{Q}_{\ell}$-пространства $V_{\ell}(X)$, коммутирующий с $K$ и $G^{\prime}$, лежит в $E_{\lambda}$. Обозначим через $M$ централизатор кольца $\mathscr{O}$ в $\operatorname{End}(X)=\operatorname{End}_{K^{\prime}}(X)$. Наша цель - доказать, что $M=\mathscr{O}$. 
Ясно, что $M$ является свободной коммутативной группой конечного ранга (по сложению), а $\mathscr{O}$ лежит в центре кольца $M$. Также ясно, что любой элемент кольца $M$, рассматриваемый как эндоморфизм в $V_{\ell}(X)$, коммутирует с $K$ и $G^{\prime}$ и, следовательно, лежит в $E_{\lambda}$. Отсюда немедленно вытекает, что

$$
E_{\lambda}=\mathscr{O} \otimes \mathbb{Q}_{\ell} \subset M \otimes \mathbb{Q}_{\ell} \subset E_{\lambda} \subset \operatorname{End}_{\mathbb{Q}_{\ell}}\left(V_{\ell}(X)\right) .
$$

Это влечет за собой равенство $\mathscr{O} \otimes \mathbb{Q}_{\ell}=M \otimes \mathbb{Q}_{\ell}$, и, следовательно свободные коммутативные группы $\mathscr{O}$ и $M$ имеют один и тот же ранг. Из включения $\mathscr{O} \subset M$ вытекает, что кольцо $M$ лежит в $\mathscr{O} \otimes \mathbb{Q}=E$. Поскольку $\mathscr{O}$-максимальный порядок в $E$ и $M \supset \mathscr{O}$, мы заключаем, что $M=\mathscr{O}$. Доказательство окончено.

\section{Список литературы}

1. Koo J. K. On holomorphic differentials of some algebraic function field of one variable over $\mathbb{C} / /$ Bull. Austral. Math. Soc. 1991. V. 43. P. 399-405.

2. Towse $C$. Weierstrass points on cyclic covers of the projective line // Trans. Amer. Math. Soc. 1996. V. 348. P. 3355-3377.

3. Poonen B., Schaefer E. Explicit descent for Jacobians of cyclic covers of the projective line // J. Reine Angew. Math. 1997. V. 488. P. 141-188.

4. Schaefer E. Computing a Selmer group of a Jacobian using functions on the curve // Math. Ann. 1998. V. 310. P. 447-471.

5. Zarhin Yu. G. Hyperelliptic Jacobians without complex multiplication // Math. Res. Lett. 2000. V. 7. P. 123-132.

6. Zarhin Yu. G. Hyperelliptic Jacobians without complex multiplication in positive characteristic // Math. Res. Lett. 2001. V. 8. P. 429-435.

7. Zarhin Yu. G. Hyperelliptic Jacobians and modular representations // Moduli of abelian varieties / ed. C. Faber, G. van der Geer, F. Oort. Basel: Birkhäuser, 2001. (Progr. Math. V. 195.) P. 473-490.

8. Zarhin $Y u$. G. Very simple 2-adic representations and hyperelliptic Jacobians // Moscow Math. J. 2002. V. 2; http://xxx.lanl.gov/abs/math.AG/0109014.

9. Zarhin $Y u$. G. Cyclic covers of the projective line, their jacobians and endomorphisms // J. Reine Angew. Math. 2002. V. 544. P. 91-110; http://xxx.lanl.gov/abs/math.AG/0003002.

10. Zarhin $Y u$. G. The endomorphism rings of Jacobians of cyclic covers of the projective line // http://xxx.lanl.gov/abs/math.AG/0103203.

11. Mumford D. Abelian varieties. London: Oxford Univ. Press, 1974.

12. Ribet $K$. Galois action on division points of Abelian varieties with real multiplications // Amer. J. Math. 1976. V. 98. P. 751-804.

13. Shimura $G$. Introduction to the arithmetic theory of automorphic functions. Princeton, NJ: Iwanami Shoten, Publ. and Princeton Univ. Press, 1971.

14. Wagner $A$. The faithful linear representations of $S_{n}$ and $A_{n}$ over a field of characteristic 2 // Math. Z. 1976. V. 151. P. 127-137.

15. Wagner $A$. Thefaithful linear representations of $S_{n}$ and $A_{n}$ over a field of odd characteristic // Math. Z. 1977. V. 154. P. 103-114.

16. Hoffman P.N., Humphreys J.F. Projective representations of the symmetric groups. Oxford: Clarendon Press, 1992.

17. Wagner A. An observation on the degrees of the symmetric and alternating group over an arbitrary field // Arch. Math. (Basel). 1977. V. 29. P. 583-589.

18. James G., Kerber $A$. The representation theory of the symmetric group. Reading, MA: Addison-Wesley, 1981. (Encyclopedia Math. Appl. V. 16.)

19. Feit W., Tits J. Projective representations of minimum degree of group extensions // Canad. J. Math. 1978. V. 30. P. 1092-1102.

20. Kleidman P. B., Liebeck M. W. On a theorem of Feit and Tits // Proc. Amer. Math. Soc. 1989. V. 107. P. 315-322.

Университет Штата Пенсильвания,

Институт математических проблем биологии РАН, Пущино

Поступила в редакцию

E-mail: zarhin@math.psu.edu

04.12 .2001 